\title{
Simultaneous intracranial and testicular germ cell tumors: illustrative case
}

\author{
Lei Han, MD, Jie Lu, MM, Luxiong Fang, MD, Songtao Qi, MD, and Ye Song, MD \\ Department of Neurosurgery, Nanfang Hospital, Southern Medical University, Guangzhou, Guangdong, People's Republic of China
}

\begin{abstract}
BACKGROUND Simultaneous intracranial and testicular germ cell tumors (GCTs) are extremely rare, leading to a lack of adequate experience in their treatment. Therefore, the authors report a case of this kind of GCT.

OBSERVATIONS A 5-year-old boy was admitted to the hospital with headache and vomiting. Computed tomography and magnetic resonance imaging suggested the possibility of a GCT in the pineal region. The value of the serum tumor marker alpha-fetoprotein (AFP) was $5,396.1 \mu \mathrm{g} / \mathrm{L}$, and $\beta$-human chorionic gonadotropin levels were within the normal range. Subsequently, the tumor was removed, and the final pathological result was a mixed GCT. Therefore, chemotherapy and radiation were added. However, the authors found a testicular tumor on ultrasound at the same time, and pathology after surgery suggested a mature cystic teratoma. Following treatment, the patient recovered well, and AFP levels dropped to normal values.

LESSONS To the authors' knowledge, this report is the fourth case of simultaneous intracranial and testicular GCTs and the first case of a simultaneous mixed GCT in the pineal region and mature teratoma of the testis. A combination of surgery, chemotherapy, and radiation therapy for mixed GCTs in the pineal region and surgical excision for testicular reproductive cell tumors are effective in these patients, but long-term monitoring is required.
\end{abstract}

https://thejns.org/doi/abs/10.3171/CASE2067

KEYWORDS simultaneous germ cell tumors; bifocal tumors; mature teratoma; mixed germ cell tumor; pineal gland; testis

Germ cell tumors (GCTs) are most often located in the gonads, though they can occur in the retroperitoneum, the mediastinum, and even the central nervous system (CNS). ${ }^{1-3}$ However, only $2 \%$ to $5 \%$ of GCTs are extragenital, ${ }^{4}$ making up a small fraction of all GCTs. Meanwhile, CNS GCTs are a rare kind of tumor, accounting for about $3-11 \%$ of brain tumors in pediatrics. ${ }^{5}$ Consequently, multiple sites of simultaneous GCTs are extremely rare, especially simultaneous intracranial and testicular GCTs. Thus, there is no uniform standard of sufficient experience for the treatment regimen. Here, we report an unusual case of a child with a simultaneous mixed GCT in the pineal gland (consisting of approximately $60 \%$ yolk sac tumor and about $40 \%$ mature cystic teratoma) and mature testicular teratoma.

\section{Illustrative Case}

The patient, a 5-year-old boy, experienced headache with vomiting as the first symptom, which resolved without treatment. However, the symptoms of headache and vomiting worsened 10 days after the onset of initial symptoms. The patient was then taken to the local hospital for treatment, and he underwent cranial computed tomography (CT) examination, which revealed an intracranial mass and hydrocephalus. A magnetic resonance imaging (MRI) examination was suggested to confirm the possibility of a GCT.

The patient had been experiencing these symptoms for half a month when he first visited our hospital. CT cross-sectional and coronal images showed irregular space-occupying lesions in the fourth ventricle and the quadrigeminal-pineal region, with clear boundaries. Moreover, the larger layer of the lesion was about $2.0 \times 3.3 \mathrm{~cm}$ (Fig. 1A). Further, a neoplastic lesion, supratentorial ventricular system dilatation, and interstitial edema were considered. The patient had no family history of cancer. He was clinically diagnosed as having a space-occupying lesion in the area of the fourth ventricle and quadrigeminal cisterna-pineal gland, considering the possibility of a GCT. Further MRI examination was conducted to confirm the possibility of a GCT, accompanied by supratentorial and interstitial hydrocephalus (Fig. 1B-D). The value of the serum tumor marker

ABBREVIATIONS AFP = alpha-fetoprotein; $\beta$-hCG = $\beta$-human chorionic gonadotropin; $C K=$ cytokeratin; $C N S=$ central nervous system; CT = computed tomography; $E+P=$ etoposide + cisplatinum; EMA = epithelial membrane antigen; GCT = germ cell tumor; GFAP = glial fibrillary acidic protein; $\mathrm{H} \& \mathrm{E}=$ hematoxylin-eosin; $\mathrm{MRI}=$ magnetic resonance imaging; OCT4 = octamer-binding transcription factor 4; PLAP = placental alkaline phosphatase; VP = ventriculoperitoneal.

INCLUDE WHEN CITING Published January 18, 2021; DOI: 10.3171/CASE2067.

SUBMITTED October 17, 2020. ACCEPTED November 2, 2020.

(C) 2021 The authors, CC BY-NC-ND 4.0 (http://creativecommons.org/licenses/by-nc-nd/4.0/) 

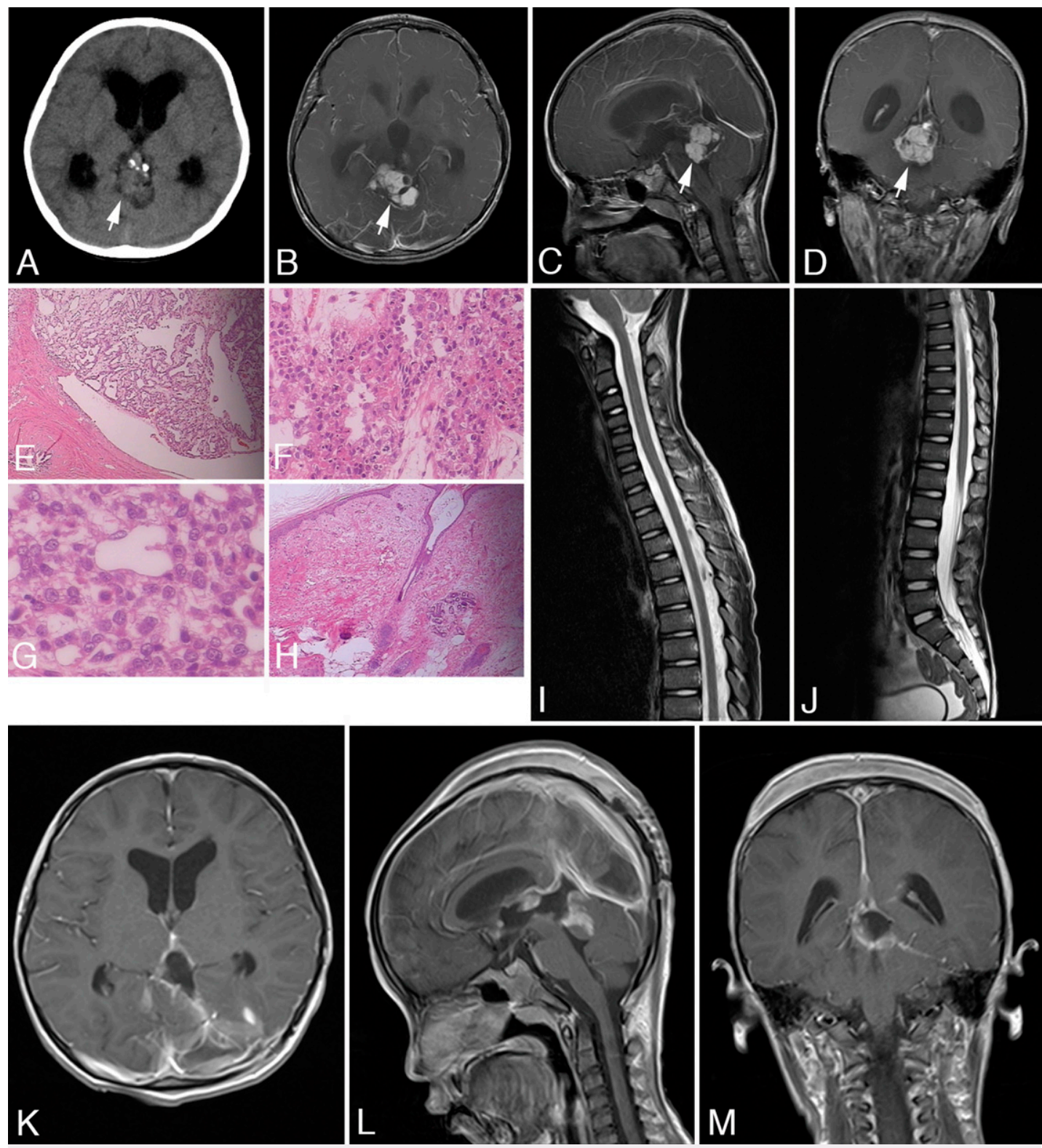

FIG. 1. A: CT examination of the brain revealed an uneven-density space-occupying lesion of $2.0 \times 3.3 \mathrm{~cm}$ in the pineal region (arrow). B-D: Contrast-enhanced MRI of the head showed a lobulated space-occupying lesion with clear boundaries in the pineal region, with the largest layer being about $2.0 \times 3.3 \mathrm{~cm}$ (arrows). E-H: H\&E staining of mixed GCTs in the pineal region. E: Original magnification $\times 100$. The tumor cells of some nodules in the tissue examined were reticular, glandular, and sac-like structures that were anastomosed to form a maze-like structure. F and G: Original magnification $\times 200$ and $\times 400$, respectively. Tumor cells are composed of flat, cubic, or columnar epithelium. They vary in size and shape, including round, quasi-round, columnar, and polygonal. Nuclear atypia is evident, and mitotic figures are visible. Some tumor tissue showed patchy necrosis. H: Original magnification $\times 100$. Some nodules are covered with squamous epithelium. There are numerous sebaceous glands, sweat glands, and follicular skin appendices. The mesenchyme is a hyperplastic fibrous connective tissue. I-J: MRI examination of the spinal cord and lumbosacral region revealed no tumor spread or metastasis. K-M: Postoperative MRI showed that the space-occupying lesion in the pineal region had obviously disappeared.

alpha-fetoprotein (AFP) was 5,396.1 $\mu \mathrm{g} / \mathrm{L}$ on the first detection. Preoperative examination was completed, and surgical contraindications were eliminated. Then the left Poppen approach was performed for a pineal gland-occupying lesion resection, dural repair, and cranioplasty in the neurosurgery unit. The gross specimens submitted for pathological examination showed a pile of grayish-white and grayish-brown broken tissue, with a volume of $4 \times 3 \times 2 \mathrm{~cm}$. Multiple enveloped nodules were seen in the specimens, with a diameter range of $0.8-2.2 \mathrm{~cm}$. The surface of the specimens was solid and grayish-white, and the nodular hardness was medium. Hematoxylin-eosin (H\&E) (Fig. 2E-H) and immunohistochemical 

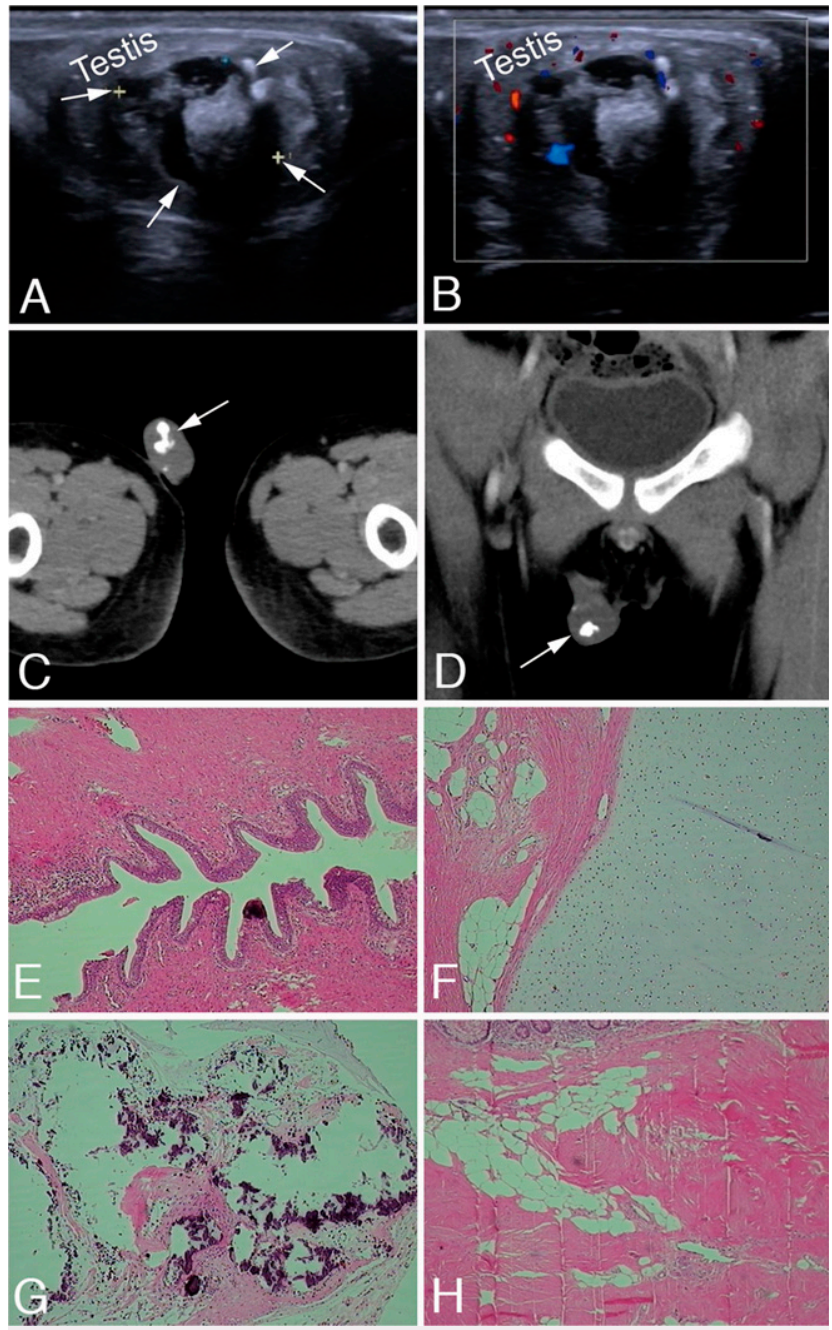

FIG. 2. A: Ultrasound examination revealed a mixed echo mass with irregular shape and unclear boundary in the right testicle, about $1.3 \times 1.0 \mathrm{~cm}$ in size (arrows). B: Color Doppler flow imaging shows the point color flow signal in the solid part of the mass. C and D: The CTexamination of the whole abdomen revealed a $2.3 \times 1.6-\mathrm{cm}$ quasi-circular space-occupying lesion with uneven density in the right testicle, and calcification can also be seen (arrows). E-H: H\&E staining of tissue specimens of testicular masses. Original magnification $\times 100$. E: The testicular tissue is covered with columnar epithelium. F: There is differentiated and mature cartilage. The mesenchyme is hyperplastic, fibrous connective tissue and adipose tissue. G: Calcification is seen in the tissue. $\mathrm{H}$ : No obvious abnormalities were found in the peripheral testis and epididymis.

staining was performed on the tissue sent for examination by the Department of Pathology of our hospital. Immunohistochemical staining results (Table 1) showed that cytokeratin (CK), AFP, and Ki67 (about 80\%) were positively expressed, whereas CD117 and placental alkaline phosphatase (PLAP) were partially positive and CK7 and epithelial membrane antigen (EMA) were positively expressed in mature glandular epithelium. In addition, glial fibrillary acidic protein (GFAP), octamerbinding transcription factor 4 (OCT4), CD30, $\beta$-human chorionic
TABLE 1. Immunohistochemical staining results of pineal region tumor

\begin{tabular}{|c|c|}
\hline & Tumor Markers \\
\hline Positive & CK, AFP, and Ki67* (about 80\%) \\
\hline Negative & $\begin{array}{c}\text { GFAP, OCT4, CD30†, } \beta \text {-hCG, D2-40‡, S-100§, } \\
\text { and oligo-2 }\end{array}$ \\
\hline Partial positivity & $\begin{array}{l}\text { CD117ף, PLAP, CK7 (mature glandular epithelium), } \\
\text { and EMA (mature glandular epithelium) }\end{array}$ \\
\hline
\end{tabular}

\author{
${ }^{*}$ Antigen Ki67. \\ $\dagger$ Human CD30. \\ $\ddagger$ Podoplanin. \\ $\S$ Calcium-binding protein. \\ ๆ Proto-oncogene c-kit.
}

gonadotropin ( $\beta$-hCG), D2-40, S-100, and oligo-2 were all negative. The final pathological diagnosis was a mixed GCT in the pineal region (about $60 \%$ for yolk sac tumor and $40 \%$ for mature cystic teratoma). Therefore, an etoposide + cisplatinum $(E+P)$ regimen was also given after the operation. Postoperative MRI examination (Fig. 1/-M) indicated a small amount of effusion in the operative area, meningeal enhancement around the operative area, and changes after resection of mixed GCTs in the pineal area. Meanwhile, supratentorial hydrocephalus and interstitial hydrocephalus were alleviated.

Considering that the tumor marker AFP was significantly higher than the normal level and the patient was younger than 6 years old, it was suggested that the patient was at high risk. Thus, we conducted a scrotal ultrasound examination, which suggested a space-occupying lesion of the right testicle (Fig. 2A and B), and the possibility of a testicular GCT was considered. There was also pelvic effusion. Therefore, CT examination of the whole abdomen was completed, which suggested a space-occupying lesion of the right testicle and pelvic effusion; therefore, the possibility of a testicular GCT was considered. Moreover, the larger layer of the focus was about $2.3 \times 1.6 \mathrm{~cm}$ (Fig. $2 \mathrm{C}$ and D). A month after the neurosurgical operation, right testicular tumor resection was performed when the patient's physical condition permitted. The pathological tissue for examination was a lump of grayish-white and grayish-red tissue about $7.5 \times 2.5 \times 1.8 \mathrm{~cm}$ in volume. The testicle was about $2.5 \times 1.8 \times 1.5 \mathrm{~cm}$ in size, and a solid mass of about $1.5 \times 1.2 \times 1.5 \mathrm{~cm}$ was observed after the testicle was cut open. The cut surface was grayish-white, and the tumor was hard and tough. H\&E staining revealed that the examined tissue was covered with columnar epithelium, and mature differentiated cartilage was seen. The mesenchyme was a hyperplastic, fibrous connective tissue and adipose tissue with calcification. There were no obvious abnormalities in the peripheral testis and epididymis. The final pathological diagnosis was a testicular mature teratoma (Fig. 2E-H).

Since the first surgery, the patient had undergone five cycles of alternate chemotherapy with E+P and ifosfamide + etoposide regimens. Radiotherapy was performed for the mixed GCTs in the pineal region during chemotherapy. MRI (Fig. 3A-C) was reviewed regularly after operation, and tumor markers were monitored. Following one month of treatment, the serum AFP level decreased significantly from the initial $5,396.1 \mu \mathrm{g} / \mathrm{L}$ to $32.2 \mu \mathrm{g} / \mathrm{L}$. In the follow-up monitoring, the serum AFP level gradually returned to the normal range (Fig. 3D). At present, the patient is recovering well, though follow-up is necessary. 

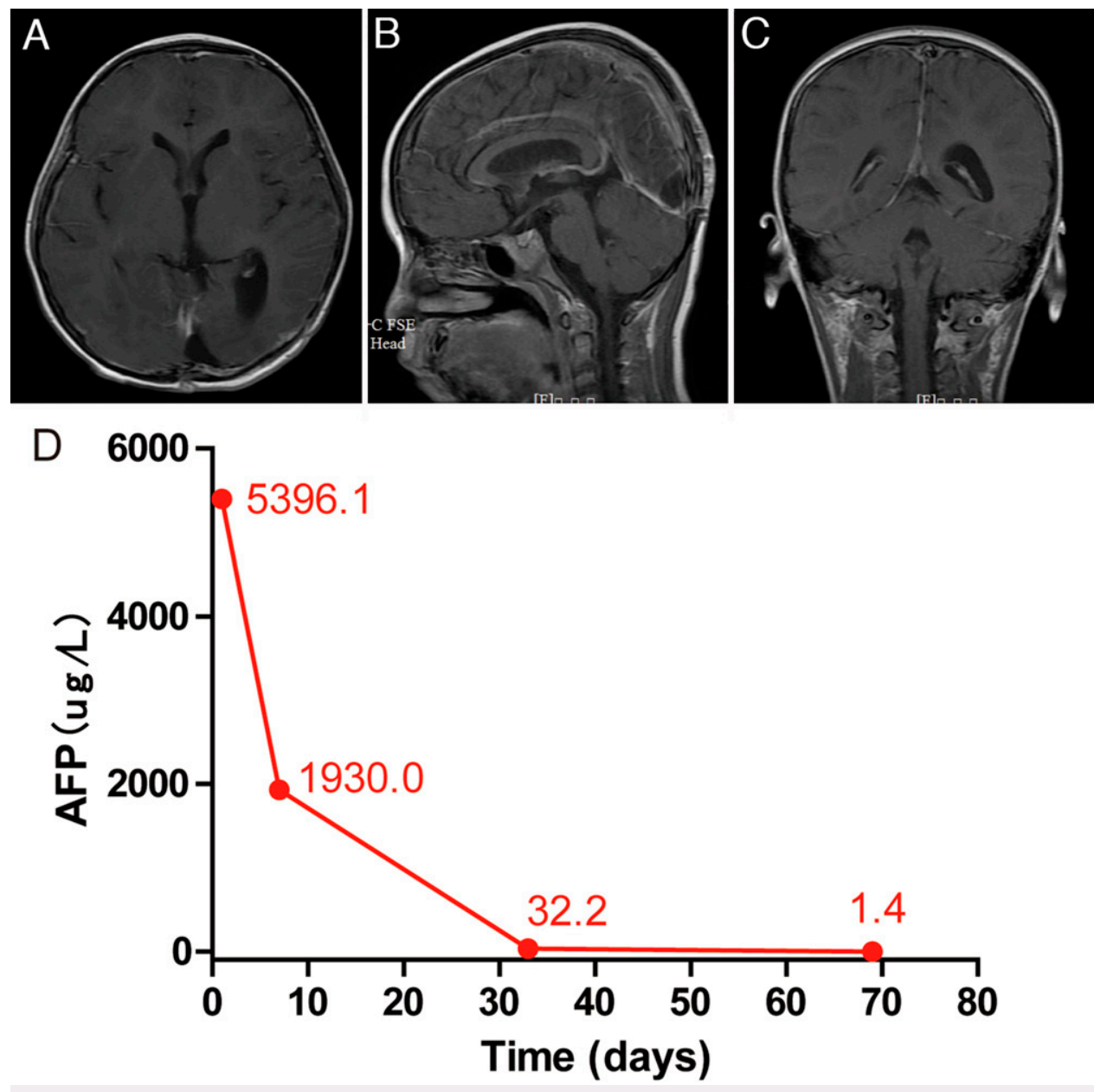

FIG. 3. A-C: Four months after the operation in the pineal region, head MRI examination showed that the operative area recovered well, and no recurrence or metastasis was found. D: Monitoring of the tumor marker AFP. The abscissa represents the time starting from the patient's first admission.

\section{Discussion}

\section{Observations}

Bifocal GCTs that occur simultaneously in different locations are rare. For intracranial GCTs, the most common bifocal GCTs are germinomas, occurring simultaneously in the pineal and suprasellar regions. They occur in $6-26 \%$ of patients with intracranial germinomas. ${ }^{6}$ In fact, we have not found such a high proportion of bifocal intracranial germinomas clinically, which may be related to the characteristics of different countries and regions. However, GCTs that occur simultaneously in the brain and testis are much rarer than bifocal intracranial germinomas. To our knowledge, only three cases of simultaneous intracranial and testicular GCTs have been reported in the literature ${ }^{7-9}$ (Table 2). Here, we report the first case in our hospital with both intracranial and testicular GCTs.

GCTs are special tumors that can theoretically occur anywhere. ${ }^{3,10}$ Also, due to the gradual onset of clinical symptoms, the tumor may have metastasized by the time that symptoms appear. Syu et al. reported a case of testicular GCT with initial symptoms of seizure and metastases to the lymph nodes, lungs, liver, and brain. ${ }^{11}$ Consequently, there are two possible etiologies for these simultaneous intracranial and testicular GCTs, including independent primary tumor and metastatic tumor. GCTs most commonly originate from the gonads, and extragonadal GCTs account for only about $3 \%$ to $7 \%$ of all GCTs. ${ }^{12}$ Therefore, a testicular neoplasm is more likely to be primary if the bifocal GCTs are considered to be caused by metastatic tumor. In one study, Patel et al. showed that brain metastases always coexisted with lung metastases in testicular GCTs. ${ }^{13}$ However, there was no tumor lesion or lymph node enlargement in the chest, mediastinum,

TABLE 2. Cases of simultaneous intracranial and testicular GCTs

\begin{tabular}{lcccc}
\hline \multicolumn{1}{c}{ Case } & Gender & $\begin{array}{c}\text { Age } \\
\text { (yrs) }\end{array}$ & $\begin{array}{c}\text { Brain (pineal } \\
\text { region) }\end{array}$ & Testis \\
\hline Hupperets et al., 19997 & Male & 21 & Germinoma & Seminoma \\
\hline Özyörük et al., 20198 & Male & 29 & Germinoma & EC \\
\hline Chan et al., 1996 & Male & 17 & Germinoma & BEC \\
\hline
\end{tabular}

$\mathrm{BEC}=$ bilateral epidermoid cyst; $\mathrm{EC}=$ embryonal carcinoma. 
abdominal cavity, or retroperitoneum on the whole-abdomen CT of our patient. At the same time, testicular pathology did not find tumor infiltration around the tumor tissue. In mature teratomas, the hemorrhagic route of metastasis is rare. Therefore, we consider that mixed GCTs in the pineal region are not metastatic from testicular teratomas. In addition, no atypia cells were found on cerebrospinal fluid examination. Metastasis of intracranial GCTs to the extraneural sites, such as the lungs and the peritoneal cavity or testis, is extremely rare, although intracranial GCTs, such as those in the pineal region, may metastasize to the lung and/or peritoneal cavity through a ventriculoperitoneal (VP) shunt. ${ }^{14,15}$ There is little possibility of spread through this route in patients with newly discovered tumors and without a VP shunt. In fact, Matsuda et al., in a retrospective study of 190 patients with GCTs of the testis or extragonadal origin, reported brain metastases in $2.6 \%$ of all patients. ${ }^{16}$ Therefore, the proportion of testicular GCTs with brain metastases is not very high. Finally, we considered this to be a primary GCT that occurs simultaneously in the pineal region and testis.

It is well known that treatment regimens for primary and metastatic tumors are different. The prognosis of testicular GCTs correlates with the site of metastasis, and patients with brain metastases tend to have the worst survival. ${ }^{13}$ However, the prognosis of patients with primary intracranial GCTs differs from that in patients with metastases. Therefore, it is helpful to determine whether the tumor is primary or metastatic for treatment and prognosis. For mature teratomas, complete resection is recommended because of their insensitivity to radiotherapy or chemotherapy. ${ }^{17,18}$ However, for mixed GCTs, a combination of multiple regimens may be required, including surgery, chemotherapy, and radiation therapy. ${ }^{17}$ For metastatic tumors, surgery is generally not required. However, in 2014, lida et al. reported the first case of testicular GCT with brain metastasis that had been successfully treated with surgery alone..$^{19}$ In our case, we considered the pineal region and testicular GCTs as separate primary tumors. Therefore, we treated the tumors at the two sites according to their respective recommended treatment regimens.

Imaging examinations, such as MRI, are important in the diagnosis of intracranial GCTs. In Wu and colleagues' special study of MRI of 85 patients with intracranial GCT, it was found that the combination of serum tumor markers and MRI was helpful in the preoperative identification of germinomas and nongerminomatous GCTs. ${ }^{20}$ Meanwhile, long-term MRI monitoring can effectively detect the recurrence of intracranial GCTs. ${ }^{21}$ Conversely, reports of metachronous tumors suggest that we should not just monitor the site of the lesion during follow-up. Rothman et al. reported the case of a patient with embryonal carcinoma of the pineal region who developed a metachronous primary testicular GCT nine years after treatment. ${ }^{22}$ Therefore, a broader, longterm imaging range should be considered during follow-up after GCT treatment.

Monitoring of tumor markers after treatment is important to reflect tumor therapeutic effect, recurrence, and/or metastasis. For example, Ohno and Narita reported a case of mixed testicular GCT in which the tumor marker $\beta$-hCG did not decrease to normal levels 8 months after surgical treatment and was finally proved to have multiple metastases ${ }^{23}$ In our case, the tumor marker AFP decreased significantly following treatment and decreased from $5,396.1 \mu \mathrm{g} / \mathrm{L}$ to $1.4 \mu \mathrm{g} / \mathrm{L}$ within the normal range after 2 months, indicating that our treatment was effective.

\section{Lessons}

In conclusion, simultaneous intracranial and testicular GCTs are extremely rare. To our knowledge, we report the fourth case of this type and the first case of simultaneous mixed GCT in the pineal region and mature teratoma of the testis. A combination of surgery, chemotherapy, and radiation therapy for mixed genital cell tumors in the pineal region and surgical excision for testicular reproductive cell tumors are effective in these patients, though long-term monitoring is required.

\section{Acknowledgments}

This work was supported by the National Nature Science Fund of China (no. 81872064), Natural Science Fund of Guangdong Province, China (no. 2020A151501122), Outstanding Youths Development Scheme of Nanfang Hospital, Southern Medical University (no. 2016J008), and National Nature Science Fund of China (no. 81760450). We would like to thank Editage for English language editing.

\section{References}

1. Peltier J, Vinchon M, Baroncini M, et al. Bifocal mixed germ-cell tumor with growing teratoma syndrome and metachronous mature metastases: case report. J Neurooncol. 2008;90(1):111-115.

2. Allaway M, Nseyo UO. Primary testicular seminoma in a patient with a history of extragonadal non-seminomatous germ cell carcinoma. Urology. 2000;55(6):949-950.

3. Scholz M, Zehender M, Thalmann GN, et al. Extragonadal retroperitoneal germ cell tumor: evidence of origin in the testis. Ann Oncol. 2002;13(1):121-124.

4. Fosså SD, Aass N, Heilo A, et al. Testicular carcinoma in situ in patients with extragonadal germ-cell tumours: the clinical role of pretreatment biopsy. Ann Oncol. 2003;14(9):1412-1418.

5. Gittleman H, Cioffi G, Vecchione-Koval T, et al. Descriptive epidemiology of germ cell tumors of the central nervous system diagnosed in the United States from 2006 to 2015. J Neurooncol. 2019;143(2):251-260.

6. Kreutz J, Potorac I, Lutteri L, et al. Adipsic diabetes insipidus revealing a bifocal intracranial germinoma. Ann Endocrinol (Paris). 2017;78(3):141-145.

7. Hupperets PS, Defesche HF, de Bruijckere LM, et al.The role of chemotherapy in intracranial germinoma: a case report. Ann Oncol. 1999;10(6):723-726.

8. Özyörük D, Kocayozgat A, Yaman-Bajin i, et al. A synchronous occurrence of bifocal intracranial germinoma and bilateral testicular epidermoid cyst in an adolescent patient with Klinefelter's syndrome. Turk J Pediatr. 2019;61(3):456-459.

9. Chan TK, Agius-Fernandez A, Bashir S, et al. Simultaneous presentation of pineal germinoma and testicular seminoma. Eye (Lond). 1996;10(Pt 5):637-639.

10. Yamada $Y$, Tomita K, Fujimura $T$, et al. Metachronous testicular tumor developing eight years after retroperitoneal extragonadal germ cell tumor. Int J Urol. 2008;15(3):267-269.

11. Syu SH, Chang CL, Shih HJ. Testicular mixed germ cell tumor presenting with seizure as the initial symptom: a case report and literature review. Int Braz J Urol. 2019;45(3):629-633.

12. Chow AK, Hoeksema J, Wang D. Playing the long surveillance ball game: metachronous testicular tumor developing after treatment and remission of extragonadal germ cell tumor. Rare Tumors. 2019;11:2036361319873196.

13. Patel HD, Singla N, Ghandour RA, et al. Site of extranodal metastasis impacts survival in patients with testicular germ cell tumors. Cancer. 2019;125(22):3947-3952.

14. Ingold B, Moschopulos M, Hutter G, et al. Abdominal seeding of an atypical teratoid/rhabdoid tumor of the pineal gland along a ventriculoperitoneal shunt catheter. Acta Neuropathol. 2006;111(1): 56-59.

15. Yang $Q$, Zhu W, Huo L. Abdominal metastases of intracranial germ cell tumor via ventriculoperitoneal shunt detected by $18 \mathrm{~F}$ FDG PET/CT. Clin Nucl Med. 2020;45(10):836-837. 
16. Matsuda Y, Tanaka T, Sato S, et al. Clinical features of patients with brain metastasis from testicular germ cell tumor. Hinyokika Kiyo. 2010;56(2):99-102.

17. Bowzyk Al-Naeeb A, Murray M, Horan G, et al. Current management of intracranial germ cell tumours. Clin Oncol (R Coll Radiol). 2018;30(4):204-214.

18. André $\mathrm{F}$, Fizazi $\mathrm{K}$, Culine $\mathrm{S}$, et al. The growing teratoma syndrome: results of therapy and long-term follow-up of 33 patients. Eur J Cancer. 2000;36(11):1389-1394.

19. lida K, Naiki T, Kawai N, et al. Metastasectomy as optimal treatment for late relapsing solitary brain metastasis from testicular germ cell tumor: a case report. BMC Res Notes. 2014;7: 865.

20. Wu CC, Guo WY, Chang FC, et al. MRI features of pediatric intracranial germ cell tumor subtypes. J Neurooncol. 2017;134(1): 221-230.

21. Cheung V, Segal D, Gardner SL, et al. Utility of MRI versus tumor markers for post-treatment surveillance of marker-positive CNS germ cell tumors. J Neurooncol. 2016;129(3):541-544.

22. Rothman J, Greenberg RE, Jaffe WI. Nonseminomatous germ cell tumor of the testis 9 years after a germ cell tumor of the pineal gland: case report and review of the literature. Can J Urol. 2008; 15(3):4122-4124.

23. Ohno M, Narita Y. A case of pathological complete remission of a brain metastasis from germ cell tumor of the testis after systemic chemotherapy. Jpn J Clin Oncol. 2010;40(12):1201.

\section{Disclosures}

The authors report no conflict of interest concerning the materials or methods used in this study or the findings specified in this paper.

\section{Author Contributions}

Conception and design: Song, Han. Acquisition of data: Han, Lu, Fang. Drafting the article: Han, Lu, Fang. Critically revising the article: Qi. Reviewed submitted version of manuscript: Han, Lu, Fang, Qi. Administrative/technical/material support: Han, Lu, Fang, Qi. Study supervision: Lu.

\section{Correspondence}

Ye Song: Nanfang Hospital, Southern Medical University, Guangzhou, Guangdong, China. songye0724@126.com. 